Pacific Journal of Mathematics

COMMUTATIVE ASSOCIATIVE RINGS AND ANTI-FLEXIBLE RINGS
H. A. ÇELIK 


\title{
COMMUTATIVE ASSOCIATIVE RINGS AND ANTI-FLEXIBLE RINGS
}

\author{
H. A. ÇELIK
}

Let $R$ be a simple anti-flexible ring of characteristic distinct from 2 and 3 . Anderson and Outcalt have proved that $R^{+}$is a commutative associative ring. The same authors have also shown that a commutative associative ring $P$ of characteristic not 2 gives rise to a simple anti-flexible ring provided $P$ has a suitably defined symmetric belinear form on it. The purpose of this paper is to give an explicit construction of such a symmetric bilinear form and determine the suitable commutative associative rings.

It is proved that for any commutative associative ring $R$, which is either free of zero divisors or a zero ring, there is a class of simple anti-flexible rings associated with $R$. It is also shown that a subclass of commutative associative rings may be used to obtain a more general class of anti-flexible rings, namely prime ones, which are not necessarily simple even if they have both of the chain conditions. Finally two important examples on certain prime antiflexible rings are given.

The results mentioned above of Anderson and Outcalt appear in [1]. In [3], Slater has shown that in semi-prime alternative rings the Nucleus and the center of an ideal of $R$ are contained in the Nucleus the center of $R$ respectively, which turns out to be very valuable in the structure theory of such rings. One of the examples shows that such results do not hold in anti-flexible rings. The other example will be of use in a later paper [2].

All algebraic structures will be of characteristic not 2. Unless mentioned otherwise the term "ring" means an anti-flexible ring which is defined by the identity

$$
(x, y, z)=(z, y, x)
$$

where $(x, y, z)=(x y) z-x(y z)$ is the associator. $R^{+}$is the ring obtained from additive group of $R$ together with the multiplication "." defined by $x y=\frac{1}{2}(x y+y x)$ for all $x, y \in R$, where $x y, y x$ are multiplications of $x$ and $y$ in $R$.

$$
\begin{aligned}
& N(R)=\{n \in R:(n, x, y)=0=(x, n, y) \text { for all } x, y \in R\} \\
& Z(R)=\{z \in N(R):[z, x]=0 \text { for all } x \in R\}
\end{aligned}
$$

are defined to be the Nucleus and the center of $R$ respectively, 
where $[z, x]=z x-x z$ is the commutator.

\section{Simple rings.}

DEFINITION 2.1. Let $R$ be a commutative associative ring, and let $\Omega$ be nonempty set such that $\Omega \cap R=\varnothing$. Define the free $\Omega$-extension of $R$ to be the commutative associative ring $R^{*}$ generated by $R \cup \Omega$ with the multiplication $p q r \cdots s t$ for the finitely many elements $p, q, r, \cdots, s, t \in R \cup \Omega$, such that the restriction of this multiplication to $R$ is the multiplication of $R$ and the identity of $R$, if it has any, is the identity of $R^{*}$. We say that $R^{*}$ is of $D$-index $n$ if

$$
d_{1} d_{2} \cdots d_{n}=0
$$

for all $d_{i} \in D, i=i, \cdots, n$, where $D$ is a subset of $\Omega$ and $n$ is a positive integer.

We should mention here that the existence of such extension of $R$ is guaranteed by the rings of polynomials over $R$ and their quotient rings for suitable ideals.

THEOREM 2.2. (i) Let $R$ be a commutative associative ring without zero divisors, or let $R$ be a zero ring. Then there exists a commutative associative ring $R^{*}$ containing $R$ and a bilinear mapping $\langle$,$\rangle of R^{*} \times R^{*}$ into $R^{*}$ such that the ring $\mathscr{R}=\left(R^{*}, \otimes\right)$ is a simple anti-flexible ring, where for $x, y \in R^{*}, x \otimes y$ is defined as $x y+\langle x, y\rangle, x y$ being the multiplication in $R^{*}$.

(ii) Let $R$ be a simple anti-flexible ring of characteristic not 3. Then for any commutative multiplication " $O$ " defined on the set $R$ such that $x^{02}=x^{2}$ for all $k \in R$, the ring $(R, o)$ is commutative and associative and there is a bilinear form on $(R, o)$ which defines $R$.

Proof. (i) (a) Assume that $R$ has no zero divisors. Suppose that $\Omega$ is a set containing a totally ordered subset $\Omega_{1}$ of at least two distinct elements. Let $R^{*}$ be the free $\Omega$-extension of $R$ of $\Omega_{1}$-index 2 . Without loss of generality, assume that $R$ has an identity element $e$, therefore $R^{*}$ has an identity element $e$. In $R^{*}$, defined a bilinear form $\langle$,$\rangle as follows:$

$\left(a_{1}\right)\langle r, s\rangle=0$ if either $r$ or $s$ belongs to the set

$$
\mathscr{S}=R \cup P \cup R P
$$

where,

$$
P=\text { the set } \Omega \backslash \Omega_{1}
$$


and the set of all finite products of elements of $\Omega \backslash \Omega_{1}$.

$\left(\mathrm{a}_{2}\right)\langle r x, s y\rangle=e=-\langle s y, r x\rangle$ if $x, y \in \Omega_{1}$ such that $x<y$ and $r, s \in \mathscr{S} \backslash\{0\}$.

$\left(\mathrm{a}_{3}\right)\langle r x, s x\rangle=0$ for all $r, s \in \mathscr{S}$ and all $x \in \Omega_{1}$. In $R^{*}$ define a new multiplication " $\otimes$ " by

$$
r \otimes t=r t+\langle r, t\rangle,
$$

and let $\mathscr{R}=\left(R^{*}, \otimes\right)$ be the ring obtained by the additive group of $R^{*}$ together with the multiplication " $\otimes$ ". In order to prove that $\mathscr{R}$ is a simple anti-flexible ring, by Theorem 3.11 of [1] it suffices to show that the bilinear form $\langle$,$\rangle satifies the following conditions:$

(1) $\langle x, x\rangle=0$,

(2) $\left\langle x^{2}, x\right\rangle=0$, for all $x \in R^{*}$,

(3) $\left\langle\left\langle R^{*}, R^{*}\right\rangle, R^{*}\right\rangle=0$,

(4) $\left\langle R^{*}, R^{*}\right\rangle \neq(0)$,

(5) $\left\langle I, R^{*}\right\rangle \varsubsetneqq I$ for any proper ideal $I$ of $R^{*}$.

It follows from $\left(a_{1}\right)$ and $\left(a_{3}\right)$ that (1) holds. To see (2), consider an arbitrary element $w$ of $R^{*}$. Since $R$ has an identity element, $w$ has the following form:

$$
w=\alpha_{0} s_{0}+\alpha_{1} s_{1} x_{1}+\alpha_{2} s_{2} x_{2}+\cdots+\alpha_{n} s_{n} x_{n}
$$

where $\alpha_{i}$ are integers, $s_{i} \in \mathscr{S}, x_{i} \in \Omega_{1}(i=1,2, \cdots, n)$ and $x_{1}<x_{2}<\cdots<x_{n}$. Then,

$$
w^{2}=\alpha_{0}^{2} s_{0}^{2}+2 \sum_{i=1}^{n} \alpha_{0} \alpha_{i} s_{0} s_{i} x_{i}
$$

So,

$$
\begin{aligned}
\left\langle w^{2}, w\right\rangle & =2 \alpha_{0} \sum_{\substack{i=1 \\
j=1}}^{n} \alpha_{i} \alpha_{j}\left\langle s_{0} s_{i} x_{i}, s_{j} x_{j}\right\rangle \\
& =2 \alpha_{0} \sum_{\substack{i=1 \\
j=1}}^{n} g_{i j} .
\end{aligned}
$$

By $\left(\mathrm{a}_{3}\right), g_{i i}$ are all zero and by $\left(\mathrm{a}_{2}\right)$

$$
g_{i j}=-g_{j i} \text { for } i \neq j \text {. }
$$

Therefore

$$
\left\langle w^{2}, w\right\rangle=0,
$$

(3) and (4) are immediate.

(5) follows from the following argument. For each proper ideal $I$ of $R^{*}$, there exists at least one element $\alpha s x$ in $I$ such that $\alpha$ is an integer, $s \in \mathscr{S}$ and $x \in \Omega_{1}$. Since $\Omega_{1}$ contains at least two distinct 
elements, the set $\left\langle I, R^{*}\right\rangle$ contains the identity element $e$, Therefore $\langle I, R\rangle \nsubseteq I$. Thus, $\mathscr{R}$ is a simple anti-flexible ring.

(b) Assume that $R$ is a zero ring. By the Zermelós well ordering axiom, the generating set $R_{1}$ of $R$ can be imbedded in a totally ordered set $\Omega_{1}$. Then consider $\Omega$ to be a set containing $\Omega_{1}$. Thus, starting with the ring (0), we obtain $R^{*}$ to which an identity element $e$ may be adjoined. To define the bilinear form $\langle$,$\rangle on R^{*}$, set the defining conditions as

$$
\left(b_{1}\right)=\left(a_{1}\right),\left(b_{2}\right)=\left(a_{2}\right),\left(b_{3}\right)=\left(a_{3}\right)
$$

with,

$$
\mathscr{S}=P \cup\{0\} \text {, where } P \text { is as in }\left(a_{1}\right) \text {. }
$$

Then an analogous proof to that of (a) shows that $\mathscr{R}=\left(R^{*}, \otimes\right)$ is a simple anti-flexible ring.

(ii) The proof of this part follows from the following argument:

Let $R$ be a ring and suppose that there is defined a commutative multiplication " $o$ " on $R$ such that $x^{2}=x^{\circ 2}$ for all $x \in R$. Then

$$
(R, o)=R^{+} \text {. }
$$

For if, $x, y \in R$, then

$$
\begin{gathered}
(x+y)^{2}=(x+y)^{\circ 2} \\
x^{2}+x y+y x+y^{2}=x^{o 2}+2 x o y+y^{o 2}
\end{gathered}
$$

or

$$
x o y=\frac{1}{2}(x y+y x) .
$$

Therefore $(R, o)=R^{+}$and is a commutative associative ring which gives rise to $R$ by the bilinear form $\langle x, y\rangle=x y-x o y$.

REMARKS. (i) The class of rings without zero divisors includes fields, integral domains, polynomial rings over such rings, group algebras of abelian groups, radical-quotient rings of commutative associative rings in which $x \neq y$ and $x y$ is nilpotent imply either $x$ is nilpotent or $y$ is nilpotent, etc.

(ii) In (a), if $R$ contains a zero divisor, then the condition (2) fails: suppose that $q \in R$, such that $q t=0$ for some $t \in R$. Then consider

$$
w=\alpha q+\beta t x_{1}+\gamma x_{2}
$$

with $\alpha, \beta, \gamma$ nonzero integers; $x_{1}, x_{2} \in \Omega_{1}$ with $x_{1}<x_{2}$. Then

$$
w^{2}=\alpha^{2} q^{2}+2 \alpha \gamma q x_{2}
$$


and,

$$
\left\langle w^{2}, w\right\rangle=-2 \alpha \beta \gamma e \neq 0 .
$$

The following corollary gives simple anti-flexible algebras of arbitrary dimension.

Corollary 2.3. Let $R=F$ be a field in Theorem (2.2) and suppose that $\Omega=\Omega_{1}$ is a totally ordered set. Then $\mathscr{R}$ is a simple anti-flexible algebra over $F$, and dimension of $\mathscr{R}$ is $\left|\Omega_{1}\right| \cdot \mathscr{R}_{\text {is }}$ associative if and only if $\left|\Omega_{1}\right|=1$.

3. Prime rings. The purpose of this section is to show that there exist various types of prime anti-flexible rings which are not simple. $R$ is prime if for any two ideals $A, B$ of $R, A B=(0)$ implies $A=(0)$ or $B=(0)$.

Proposition 3.1. Let $R$ be a commutative associative ring generated by a totally ordered set $R_{1}$ which contains at least two distinct elements. Suppose that $x y=y x=0$ for all distinct $x, y \in R_{1}$, and $x^{2}=0$ for all $x \in R_{1}$, except for a fixed $z \in R_{1}$, in which case the $z^{n \prime} s$ are all distinct for $n \geqq 1$. Then, there exists a prime anti-flexible, not simple ring $\mathscr{R}$ based on $R$.

Proof. Let $\Omega$ be a nonempty set such that $\Omega \cap R=\varnothing$. Let $R^{*}$ be the free $\Omega$-extension of $R$. Consider the set

$$
\mathscr{S}=P \cup\left\{z^{n}\right\}_{n \geqq 2} \cup P\left\{z^{n}\right\}_{n \geqq 2}
$$

and a fixed element $a \in \Omega$, where $P$ is the set $\Omega$ and the set of finite products of elements of $\Omega$. Define a bilinear form in $R^{*}$ by

(a) $\langle r, s\rangle=0$ if $r$ or $s \in \mathscr{S}$

(b) For any $x, y \in R_{1}$, if $x<y$, then

$$
\begin{aligned}
& \langle x, y\rangle=\langle g x, y\rangle=\langle x, h y\rangle=\langle g x, h y\rangle=a \\
& \langle y, x\rangle=\langle y, g x\rangle=\langle h y, x\rangle=\langle h y, g x\rangle=-a
\end{aligned}
$$

for all $g, h \in P$.

(c) $\langle g x, h x\rangle=0=\langle x, x\rangle=\langle g x, x\rangle=\langle x, h x\rangle$ for all $g, h \in P$ and all $x \in R_{1}$.

Then for $r, s \in R^{*}$, define

$$
r \otimes s=r s+\langle r, s\rangle .
$$

It is not difficult to verify that the bilinear form has the properties (1)-(4) mentioned in the proof of Theorem (2.2). Therefore $\mathscr{R}=$ 
$\left(R^{*}, \otimes\right)$ is an anti-flexible ring. $\mathscr{R}$ is not simple because $a \in \Omega$ generates a proper ideal of $\mathscr{R}$. To see this we observe that $a \neq 0$ and any $x \in R_{1}$ does not belong to this ideal. Similarly, each $z^{n}$ for $n \geqq 2$ generates a proper ideal of $\mathscr{R}$. In any case, each ideal contains a finite sum of elements of the form $\alpha_{i} p_{i} z^{n_{i}}$ for $n_{i} \geqq 1, \alpha_{i}$ are integers and $p_{i} \in P$. It is clear that the product of any two elements in $\mathscr{R}$ of this type is not zero whenever both of them are not zero. Thus $\mathscr{R}$ is a prime ring.

CoRollary 3.2. In Proposition (3.1), let $R$ be a zero algebra generated by a finite set $R_{1}$ of at least two distinct elements, over a field $F$. Suppose that $\Omega=\{a\}$. Then $\mathscr{R}$ is a prime, anti-flexible, not simple algebra over $F$. Moreover, $\mathscr{R}$ has both of the chain conditions on ideals.

Proof. Suppose that $R_{1}=\left\{x_{1}, x_{2}, \cdots, x_{n}\right\}$ with the natural ordering $x_{1}\left\langle x_{2}\left\langle\cdots x_{n}\right.\right.$. If we define the bilinear form $\langle$,$\rangle as in the$ Proposition (3.1), then, $\mathscr{R}=\left(R^{*}, \otimes\right)$ is an anti-flexible algebra based on $R . \mathscr{R}$ is prime because any ideal of $\mathscr{R}$ contains the element $a$, and $a \otimes a=a^{2} \neq 0 . \quad \mathscr{R}$ is not simple since $a$ generates a proper ideal of $\mathscr{R}$. $\mathscr{R}$ has both of the chain conditions on ideals, because the only proper ideals of $\mathscr{R}$ are the ideals generated by the proper subsets of

$$
\left\{x_{1}, x_{2}, \cdots, x_{n} ; \alpha\right\}
$$

COROLlary 3.3. There exist finite dimensional anti-flexible algebras which are prime but not simple.

Proof. Suppose that $R$ is as in Corollary (3.2), and $\Omega=\{a=$ $\left.w_{1}, w_{2}, \cdots, w_{m}\right\}$. It is possible to construct $R^{*}$ in such a way that for each $i=1, \cdots, m$, there exists a positive integer $n_{i} \geqq 2$ such that $w_{i}^{n_{i}}=w_{i}$. Then, defining the bilinear form $\langle$,$\rangle as in Propo-$ sition (3.1), $\mathscr{R}$ becomes a prime anti-flexible but not simple algebra over $F$. The fact that $\mathscr{R}$ is finite dimensional is an easy consequence of the conditions imposed on elements of $\Omega$ and the finiteness of both $R_{1}$ and $\Omega$.

REMARK. The type of commutative associative rings which are used in Proposition (3.1) can easily be found as follows:

Let $Q$ be a zero ring generated by a totally ordered set $Q_{1}$. Consider $Q[z]$, the ring of polynomials in $z$. Let $Q[z]_{2}$ be the ring of $2 \times 2$ matrices on $Q[z]$. Set 


$$
R_{1}=\left\{\bar{z}=\left(\begin{array}{ll}
z & 0 \\
0 & 0
\end{array}\right) \text {, and } \bar{s}=\left(\begin{array}{ll}
0 & 0 \\
0 & s
\end{array}\right): \in Q_{1}\right\} \text {. }
$$

Let $R$ be the subring of $Q[z]_{2}$ generated by the set $R_{1}$. Then $R$ has the required properties.

\section{Two examples.}

Proposition 4.1. There exists an anti-flexible ring $R$ such that both $R$ and $R^{+}$are prime.

Proof. Let $R$ be the free commutative associative ring generated by a totally ordered set $S$ of at least three elements. Let $I$ be the ideal of $R$ generated by monomials of degree two or more in $S$. On $R$ define a bilinear form $\langle$,$\rangle as follows:$

(a) $\langle r, s\rangle=0$ if $r$ or $s$ belong to the set $\{a, I\}$ where $a$ is a fixed element of $S$.

(b) $\langle x, y\rangle=a=-\langle y, x\rangle$ if $x, y \in S \backslash\{a\}$ and $x<y$.

(c) $\langle x, x\rangle=0$ for all $x \in S$.

Then the conditions $(\mathrm{a})=(\mathrm{c})$ satisfy the properties (I) - (IV) of the proof of Theorem (2.2), with $R^{*}=R$, and hence $\mathscr{R}=(R, \otimes)$ with $r \otimes s=r s+\langle r, s\rangle$ becomes an anti-flexible ring. It follows from (a) - (c) that any ideal of $\mathscr{R}$ must contain elements of the form $a+p$ with $p \in I$. Since for $p, q \in I$

$$
(a+p) \otimes(a+q)=a^{2}+a q+p a+p q \neq 0
$$

$\mathscr{R}$ is prime. To see that $\mathscr{R}^{+}$is also prime, we observe that $\mathscr{R}^{+}$ has no nonzero divisors of zero, because for any $r, s \in \mathscr{R}$,

$$
\begin{aligned}
(r, s)_{\otimes} & =\frac{1}{2}(r \otimes s+s \otimes r) \\
& =r s=0
\end{aligned}
$$

if and only if one of $r, s$ is 0 .

\subsection{Nucleus and the Center of Ideals.}

Given $R$ and a proper ideal $A$ of $R$, the following inclusions are hoped to hold:

$$
\begin{aligned}
& N(A) \subseteq N(R) \\
& Z(A) \leqq Z(R) .
\end{aligned}
$$

In semi-prime alternative rings these inclusions hold [3] and are very useful in the related structure theory [4], [5]. It is unfortunate that the same results do not hold for the class of anti-flexible 
rings.

EXAMPLE. Let $\mathscr{R}$ be the ring obtained by Proposition (3.1), and let $I$ be the ideal generated by $z^{n}$, for some $n \geqq 2$. $I$ is a proper ideal of $\mathscr{R}$. Since $z^{n} \in \mathscr{S}, z^{n} \otimes r=z^{n} r$ for every $r \in \mathscr{R}$. Therefore $I$ is a commutative associative ring and hence

$$
N(I)=I \text { and } Z(I)=I \text {. }
$$

On the other hand $N(R)=(0)=Z(R)$. To see this consider any $x, y \in R$, with $x<y$ and $b \in \mathscr{S}$. By the construction of $R^{*}$, if $s_{1}, s_{2} \in \mathscr{S}$ then $s_{1} s_{2}$ is distinct from both $s_{1}$ and $s_{2}$. Following this argument and calculating the associator $(x, y, b)_{\otimes}$ we get

$$
\begin{aligned}
(x, y, b)_{\otimes} & =(x \otimes y) \otimes b-x \otimes(y \otimes b) \\
& =\langle x, y\rangle b+\langle b, y\rangle x-\langle x b, y\rangle \\
& =a b-a \neq 0 .
\end{aligned}
$$

This implies that neither $x, y$ of $R_{1}$ nor $b$ of $\mathscr{S}$ can be in the nucleus of $\mathscr{R}$. Therefore,

$$
N(R)=(0)
$$

Thus

$$
N(I) \nsubseteq N(R)
$$

and

$$
Z(I) \nsubseteq Z(R) \text { • }
$$

REMARK. In this paper the term "simple" is relaxed up to the ideals which are integer multiples of $R$.

\section{REFERENCES}

1. C. T. Anderson, D. L. Outcalt, On simple anti-flexible rings, J. Algebra, 10 (1968), 310-320.

2. H. A. Çelik, On primitive and prime anti-flexible rings, (to appear in J. Algebra).

3. M. Slater, Ideals in semi-prime alternative rings, J. Algebra, 8 (1968), 60-76.

4. - Prime alternative rings I, J. Algebla, 15 (1970), 229-243.

5. —- Prime alternative rings II, J. Algebla, 15 (1970), 244-251.

Received December 1, 1970. This paper is part of the author's 1971 University of California, Santa Barbara dissertation. The author would like to thank his advisor Professor D. L. Outcalt for his guidance and encouragement. This work was supported in part by the U.S. Air Force Office of Scientific Research under Grant AFOSR 698-67. 


\section{PACIFIC JOURNAL OF MATHEMATICS}

\section{EDITORS}

\author{
H. SAMELSON \\ Stanford University \\ Stanford, California 94305 \\ C. R. HовBY \\ University of Washington \\ Seattle, Washington 98105
}

J. DUGUndis

Department of Mathematics

University of Southern California

Los Angeles, California 90007

RICHARD ARENS

University of California

Los Angeles, California 90024

\section{ASSOCIATE EDITORS}
E. F. BeCKenbaCH
B. H. NeUmanN
F. WOLF
K. YoshidA

\section{SUPPORTING INSTITUTIONS}

\author{
UNIVERSITY OF BRITISH COLUMBIA \\ CALIFORNIA INSTITUTE OF TECHNOLOGY \\ UNIVERSITY OF CALIFORNIA \\ MONTANA STATE UNIVERSITY \\ UNIVERSITY OF NEVADA \\ NEW MEXICO STATE UNIVERSITY \\ OREGON STATE UNIVERSITY \\ UNIVERSITY OF OREGON \\ OSAKA UNIVERSITY \\ UNIVERSITY OF SOUTHERN CALIFORNIA
}

\author{
STANFORD UNIVERSITY \\ UNIVERSITY OF TOKYO \\ UNIVERSITY OF UTAH \\ WASHINGTON STATE UNIVERSITY \\ UNIVERSITY OF WASHINGTON

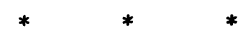 \\ AMERICAN MATHEMATICAL SOCIETY \\ CHEVRON RESEARCH CORPORATION \\ NAVAL WEAPONS CENTER
}

The Supporting Institutions listed above contribute to the cost of publication of this Journal, but they are not owners or publishers and have no responsibility for its content or policies.

Mathematical papers intended for publication in the Pacific Journal of Mathematics should be in typed form or offset-reproduced, (not dittoed), double spaced with large margins. Underline Greek letters in red, German in green, and script in blue. The first paragraph or two must be capable of being used separately as a synopsis of the entire paper. The editorial "we" must not be used in the synopsis, and items of the bibliography should not be cited there unless absolutely necessary, in which case they must be identified by author and Journal, rather than by item number. Manuscripts, in duplicate if possible, may be sent to any one of the four editors. Please classify according to the scheme of Math. Rev. Index to Vol. 39. All other communications to the editors should be addressed to the managing editor, Richard Arens, University of California, Los Angeles, California, 90024.

50 reprints are provided free for each article; additional copies may be obtained at cost in multiples of 50 .

The Pacific Journal of Mathematics is published monthly. Effective with Volume 16 the price per volume (3 numbers) is $\$ 8.00$; single issues, $\$ 3.00$. Special price for current issues to individual faculty members of supporting institutions and to individual members of the American Mathematical Society: $\$ 4.00$ per volume; single issues $\$ 1.50$. Back numbers are available.

Subscriptions, orders for back numbers, and changes of address should be sent to Pacific Journal of Mathematics, 103 Highland Boulevard, Berkeley, California, 94708.

PUBLISHED BY PACIFIC JOURNAL OF MATHEMATICS, A NON-PROFIT CORPORATION

Printed at Kokusai Bunken Insatsusha (International Academic Printing Co., Ltd.), 7-17, Fujimi 2-chome, Chiyoda-ku, Tokyo, Japan. 


\section{Pacific Journal of Mathematics}

Vol. 38, No. $2 \quad$ April, 1971

Richard Davis Anderson and Thomas Ashland Chapman, Extending

homeomorphisms to Hilbert cube manifolds .................. 281

Nguyen Huu Anh, Restriction of the principal series of $\operatorname{SL}(n, \mathbf{C})$ to some

reductive subgroups................................ 295

David W. Boyd, Indices for the Orlicz spaces . . . . . . . . . . . . 315

William Garfield Bridges, The polynomial of a non-regular digraph ...... 325

Billie Chandler Carlson, Robert K. Meany and Stuart Alan Nelson, Mixed

arithmetic and geometric means........................ 343

H. A. Çelik, Commutative associative rings and anti-flexible rings ...... 351

Hsin Chu, On the structure of almost periodic transformation groups ...... 359

David Allyn Drake, The translation groups of n-uniform translation

Hjelmslev planes ................................ 365

Michael Benton Freeman, The polynomial hull of a thin two-manifold . . . . 377

Anthony Alfred Gioia and Donald Goldsmith, Convolutions of arithmetic

functions over cohesive basic sequences .................... 391

Leslie C. Glaser, A proof of the most general polyhedral Schoenflies

conjecture possible ................................

Thomas Lee Hayden and Ted Joe Suffridge, Biholomorphic maps in Hilbert

space have a fixed point ................................ 419

Roger Alan Horn, Schlicht mappings and infinitely divisible kernels ...... 423

Norman Ray Howes, On completeness ...................... 431

Hideo Imai, Sario potentials on Riemannian spaces................ 441

A. A. Iskander, Subalgebra systems of powers of partial universal

algebras.

Barry E. Johnson, Norms of derivations of $\mathscr{L}(\mathrm{X})$.

David Clifford Kay and Eugene W. Womble, Axiomatic convexity theory and relationships between the Carathéodory, Helly, and Radon numbers

Constantine G. Lascarides, A study of certain sequence spaces of Maddox

and a generalization of a theorem of Iyer .............

C. N. Linden, On Blaschke products of restricted growth .

John S. Lowndes, Some triple integral equations ................. 515

Declan McCartan, Bicontinuous preordered topological spaces ......... 523

S. Moedomo and J. Jerry Uhl, Jr., Radon-Nikodým theorems for the Bochner and Pettis integrals ...

Calvin Cooper Moore and Joseph Albert Wolf, Totally real representations

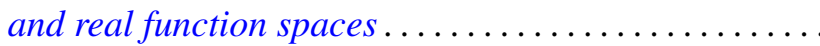

Reese Trego Prosser, A form of the moment problem for Lie groups. ... 